\title{
Conformational Polymorphism on Styrl Quinolinium Salts of 2-[(E)-2- (4-Hydroxy-3-methoxystyrl)-1-methyl]-quinolinium 4-Chlorobenzenesulfonate (HMQ-CBS): Potential Nonlinear Optical Crystals for Terahertz Application
}

\author{
Maruthappan Manikandan, Tianliang Chen, Zhihua Sun,* Shuquan Zhang, Junhua Luo*
}

Key Laboratory of Optoelectronic Materials Chemistry and Physics, Fujian Institute of Research on the Structure of Matter, Chinese Academy of Sciences, Fuzhou, Fujian 350002, China

Corresponding email: sunzhihua@fjirsm.ac.cn (Z. Sun); jhluo@ fjirsm.ac.cn (J. Luo)

\begin{abstract}
:
Polymorphism is an important phenomenon to investigate the relationship between the chemical structures and properties of functional materials. Here, we demonstrate the existence of conformational polymorphism in the terahertz (THz) compounds of 2-[(E)-2(4-hydroxy-3-methoxystyrl)-1-methyl]-quinolinium 4-chlorobenzenesulfonate (abbreviated as HMQ-CBS). There are two different crystalline phases (I and II, respectively). X-ray structural analyses reveal that HMQ-CBS crystallizes in the monoclinic crystallographic system with the non-centrosymmetric space group $P_{\mathrm{c}}$ for phase-I, and centrosymmetric space group of $P 2_{1 / n}$ for phase-II. These two different crystalline phases exhibit the distinct anionic orientations and crystal packings, leading to significantly different nonlinear optical (NLO) properties. Second harmonic generation (SHG) measurements reveal that crystals in phase-I possess the SHG efficiency of about 0.5 times that of $N, N$-dimethylamino- $N$ '-methylstilbazolium $p$-toluenesulphonate (DAST), which indicates that HMQ-CBS in phase-I might be a potential NLO crystal for THz application. In contrast, crystals in the phase-II show no SHG responses. Comparison of their crystal structures and NLO properties enables us to understand the correlation between molecular conformational changes and bulk NLO properties, and sheds light on the design of organic THz materials.
\end{abstract}

Keyword: Polymorphism, Nonlinear optics, THz crystal, Molecular packing

\section{Introduction:}

Crystalline polymorphism has been considered as a fascinating research area because polymorphs exhibit different mechanical, physical and chemical properties, which makes it great importance in pharmaceutical and material science fields [1-2]. Particularly, the engineering of new materials with desirable functional properties require the knowledge of structure-property relationship, which could be achieved by investigating the polymorphism systems. In organic polymorphisms, the structure-property relationship is directly dependent on the molecular conformations and the packing arrangements. Therefore, organic molecular compounds have great 
possibilities to exhibit the polymorphism because of their flexibility and weak intermolecular interactions in the solid state [3-7].

Styrylquinolinium derivatives with methylquinolinium electron acceptors are recently found to be interesting materials for nonlinear optical (NLO) applications, due to their higher electron accepting strength than the methylpyridinium acceptor in the benchmark ionic NLO crystal of $N, N$-dimethylamino- $N$ '-methylstilbazolium-4-methylbenzenesulfonate (DAST, one of the most promising crystals for terahertz application) [8]. Quinolinium-based crystals also exhibit large microscopic and macroscopic NLO susceptilibilities [8-10]. Moreover, such quinolinium crystals show a high terahertz (THz) wave generation efficiency at near infrared and infrared wavelengths [11-12]. Interestingly, polymorphism is quite common in the stilbazolium and quinolinium derivatives. For instance, S.H. Lee et al. had reported the crystalline polymorphism of 2-[(E)-2-(4-hydroxy-3-methoxystyrl)-1-methyl]-quinolinium 4-methoxybenzenesulfonate, that is, two different crystalline states of $\alpha$-phase and $\beta$-phase. These polymorphs display the different macroscopic second-order NLO responses. In detail, the effective hyperpolarizability tensor component $\beta_{i j k}^{e f f}$ of $\alpha$-phase crystals is the diagonal component $\beta_{333}^{e f f}=178 \times 10^{-30} e s u$, while the corresponding value for $\beta$-phase crystals is the off-diagonal component $\beta_{223}^{e f f}=60 \times 10^{-30}$ esu [13]. However, the investigation on polymorphism of quinolinium derivatives is rather rare, and the relationship between crystal structures and NLO properties has not yet been fully investigated.

In the present work, we report the polymorphism of the NLO molecular compound, 2-[(E)-2(4-hydroxy-3-methoxystyrl)-1-methyl]-quinolinium 4-chlorobenzenesulfonate (HMQ-CBS), which exhibit two different crystalline states (phase I and phase II) determined by X-ray structural analyses and second harmonic generation (SHG) measurements. The results of NLO properties display that crystals in the phase-I possess the SHG efficiency of about 0.5 times that of DAST, indicating that it might be a potential NLO crystal for THz application. In contrast, crystals in the phase-II show no SHG responses. Comparison of their crystal structures and NLO properties enables us to understand the correlation between molecular conformations and bulk NLO properties, which will shed light on the further studies of organic $\mathrm{THz}$ materials.

\section{Experimental section:}

Metathesization of 2-[(E)-2-(4-hydroxy-3-methoxystyrl)-1-methyl]-quinolinium iodide with the silver-4-chlorobenzensulfonate in hot methanol afforded the raw materials of HMQ-CBS [14-15]. The synthesis of iodide precursor was described in the literature including condensation of dimethylquinolinium iodide with vanillin in the presence of piperidine. Subsequently, the synthesized HMQ-CBS materials were recrystallized for multiple times from methanol solutions.

In order to investigate the crystal structures of HMQ-CBS crystals, single crystals were grown by the evaporation method from methanol and methanol-acetonitrile mixed solvents, respectively. According to the crystal growth procedure reported in the literature, [14] need-like orange color 
crystals were obtained from methanol solutions, which crystallize in the non-centrosymmetric space group of $P_{\mathrm{c}}$ (phase-I, see the following X-ray structure determination). Moreover, dark red color crystals of another phase (phase-II, the centrosymmetric space group of $P 2_{1} / n$ ) were obtained from the mixed methanol-acetonitrile solutions. X-ray powder diffraction (XRPD) patterns were collected to confirm their bulk purities, and compared with the simulated results from single-crystal structures, respectively (as shown in Figures S1 and S2).

\section{Results and discussion:}

Single-crystal X-ray structure determination reveals that both phases of HMQ-CBS crystals crystallize in monoclinic crystal system. However, phase-I belong to the non-centrosymmetric space group of $P_{\mathrm{c}}$ and phase-II has the centrosymmetric space group of $P 2_{1 /} n$. Crystal structure of phase I has been recorded in the Cambridge Structural Database [14]. Here, we cited the reported structure from the reference for a consistent comparison.

Firstly, it is noteworthy that molecular conformation of the organic $\pi$-conjugated cations differentiates the two polymorphs of HMQ-CBS. Fig. 1a shows that the organic cation of phase-I is almost planar with a dihedral angle of $4.2^{\circ}$ between the quinolinium part and benzene rings. The benzene ring of chlorobenzenesulfonate anion affords the dihedral angles of $80.07^{\circ}$ and $84.24^{\circ}$, respectively, with the quinolinium part and benzene rings of the cation. In contrast, the organic cation of phase-II undergoes an obvious conformational twisting and thus displays a non-planar molecular configuration. Such a change is solidly reduced from the dihedral angle of $\sim 14.81^{\circ}$, which results from steric hindrance between hydrogen atoms on the $\mathrm{N}$-substituted methyl group of quinolinium part and hydrogen atoms on the $\mathrm{C}=\mathrm{C}$ group [8]. Torsion angles of the quinolinium with respect to the cationic benzene ring also confirm this conformational twisting (see Table S1). As a result, this large conformational twisting in phase-II induces the orientation difference for chlorobenzenesulfonate anion as compared to phase-I, and finally results in the formation of polymorphs.

Moreover, these two phases also exhibit distinguishing assignment of hydroxy groups in the phenyl ring. For phase II, the hydroxy group faces towards the neighboring methoxy group in the phenyl, forming strong intramolecular hydrogen bonding between the hydroxy group and methoxy group with distances of about $2.44 \AA$ within the cation. While in the case of phase-I, it appears in opposite direction to the methoxy group. In many cases, the direction of the hydroxy group could strongly influence the molecular first hyperpolarizability of the NLO crystals [16]. This noticeable difference in the orientation of hydroxy groups may be ascribed to the different alignment of counterion with cation in the molecular structure. Furthermore, intermolecular hydrogen bonds between hydroxy group and sulfonate group also different for two phases. Above results disclose that the polymorphs of HMQ-CBS crystals contain different molecular conformers of organic cations and hydrogen-bonding interactions, which might create the different crystal packing arrangements. 
The most striking feature is that two polymorphs of HMQ-CBS crystals exhibit different packing motifs, as shown in the Figures $1 \mathrm{~d}$ and $2 \mathrm{c}$. All the molecules pack into a single-layer structure for phase-I, of which the cationic and ionic moieties are stacked alternatively along the $c$-axis. Particularly, the perfectly parallel alignment of the cations with the perpendicular anionic planes might be favorable for the non-centrosymmetric packing, as well as the NLO responses. However in phase-II, the molecules pack into the two-layer structures with an antiparallel alignment of two active cation-anion pairs. Even though the cationic plane and anionic moiety are almost parallel to each other, owing to the antiparallel dipole alignments between the cation-anion pairs, crystal of phase-II is packed into a centrosymmetric structure.

Thermal stability of HMQ-CBS crystals of phase-I was studied by the thermogravimetric (TG)

and differential thermal (DTA) analyses. The results indicate that HMQ-CBS crystals start decomposing around $190^{\circ} \mathrm{C}$ and the residue beomes melted at $\sim 255^{\circ} \mathrm{C}$, which well corresponds to the endothermic peak in the DTA trace (Figure S4).

SHG activities of two polymorphs were investigated by Kurtz and Perry powder technique using Q-switching laser with the wavelength of $2.0 \mu \mathrm{m}$ as the fundamental radiation beam. The non-resonant light with second-harmonic wavelength $(\sim 1.0 \mu \mathrm{m})$ was observed with a precise photodiode, which was quite resistive to the fundamental wavelength. An appropriate filter was settled for eliminating the contributions of the potential third-harmonic generation $(\lambda=683 \mathrm{~nm})$. $[17,18]$ The oscilloscope photoelectric signals reveal that phase-I has SHG efficiency of about $\sim 0.5$ times that of DAST (Figure 3), approximately equal to 500 times that of urea. Such an observation discloses that crystals in phase-I may be a potential NLO candidate for THz application. However, the crystals of phase-II display no significant SHG activities. The absence of SHG signals for phase-II also fairly agrees with the centrosymmetric crystallographic structure. The noteworthy difference of NLO activities between two polymorphs results from the differences of molecular conformational in their crystal structures.

\section{CONCLUSION:}

Conformational polymorphism of the NLO crystals HMQ-CBS has been investigated, and a new polymorph was found with different anionic orientation and molecular arrangement. Both phases of HMQ-CBS crystals belong to the monoclinic system, but show different space groups (i.e. $P c$ and $P 2_{1} / n$ ). The remarkable diversity between the two polymorphs of HMQ-CBS has been related to the molecular conformational changes, which influences the anionic orientation, crystal structure and the bulk NLO properties. Particularly, crystals in phase-I exhibit the SHG efficiency of about 0.5 times that of DAST, which may be a potential NLO candidate for THz application These results will lead the promising way to design new NLO materials for the THz application and to understand the structure-property relationship in organic functional materials. 


\section{ACKNOWLEDGEMENTS:}

This work was supported by NSFC (21222102, 21373220, 51402296, 21171166, 21301172 and 21450110415), the 973 Key Program of MOST (2011CB935904), the NSF for Distinguished Young Scholars of Fujian Province (2014J06015), the "Chunmiao Project" of Haixi Institute of Chinese Academy of Sciences (CMZX-2013-002), the Youth Innovation Promotion of CAS (2014262), and the CAS President's International Fellowship Initiative.

\section{REFERENCES:}

[1]. E. S. Ferrari, R. J. Davey, Cryst. Growth Des., 4 (2004) 1061-1068.

[2]. Z. Guoqi, Cryst.Eng.Comm., 15 (2013) 6453-6456.

[3]. J. Bernstein, R. J. Davey, J. O. Henck, Concomitant polymorphs. Angewandte Chemie International Edition 38 (1999) 3440-3461.

[4]. N. Blagden, R. J. Davey, Cryst. Growth Des., 3 (2003) 873-885.

[5]. M.R. Caira, Crystalline polymorphism of organic compounds. Topics in Current Chemistry 198 (1998) 164-208.

[6]. J. Bernstein, J. Phys. D: Appl. Phys., 26 (1993) B66-B76.

[7]. T. V. Timofeeva, V. N. Nesterov, F. M. Dolgushin, Y. V. Zubavichus, J. T. Goldshtein, D. M. Sammeth, et al. Crystal Engineering, 3 (2000) 263-288.

[8]. P. J. Kim, T. H. Jeong, M. Jazbinsek, S. B. Choi, I. H. Baek, J. T. Kim, et al., Adv. Func. Maters., 22 (2012) 200 - 209.

[9]. S. Chantrapromma, B. Jindawong, H. K. Fun, P. S. Patil, Analytical Sciences, 23 (2007) 81 -82 .

[10]. P. J. Kim, M. Jazbinsek, J. H. Jeong, J. T. Kim, Y. S. Lee, Y. M. Jung, et al., Cryst. Eng. Comm., 14 (2012) 3633 - 3637.

[11]. J. H. Jeong, B. J. Kang, J. S. Kim, M. Jazbinsek, S. H. Lee, S. C. Lee, et al., Sci. Rep. 3 (2013) 3200-1-3200-8.

[12]. S. H. Lee, B. J. Kang, J. S. Kim, B. W. Yoo, J. H Jeong, K. H. Lee, et al., Adv. Opt. Mater., 3 (2015) 756-762.

[13]. S. H. Lee, M. Jazbinsek, H. Yun, J. T. Kim, Y. S. Lee, O. P. Kwon, Dyes and Pigments, 96 (2013) 435 - 439.

[14]. S. Chantrapromma, B. Jindawong, H. K. Fun, P. S. Patil, C. Karalai, Anal. Sci. X-ray Struct. Anal. Online, 23 (2007) x27 - x28.

[15]. S. Chantrapromma, B. Jindawong, H. K. Fun, P. S. Patil, C. Karalai, Acta Cryst., E62 (2006) o1802- o1804. 
[16]. O. P. Kwon, M. Jazbinsek, J. I. Seo, E.Y. Choi, H. Yun, F. D. J. Brunner, P. Gunter, Y. S. Lee, J. Chem. Phys., 130 (2009) 134708-1-7.

[17] Z. Sun, X. Liu, X. Wang, L. Li, X. Shi, S. Li, . Ji, J. Luo, M. Hong, Cryst. Growth Des., 12 (2012) 6181-6187.

[18] Z. Sun, T. Chen, J. Luo, M. Hong, J. Cryst. Growth, 2011, 328, 89-94.

Figure 1: Scheme and crystal structures of HMQ-CBS in phase-I. (a) schematic diagram of HMQ-CBS; (b) Molecular conformation of the cationic moiety; (c) molecular structure; and (d) crystal packing diagram viewed along $b$-axis. Hydrogen bonds are depicted as the dash lines.

Figure 2: Crystal structures of HMQ-CBS in phase-II. (a) Molecular conformation of the cationic moiety; (b) molecular structure, and (c) crystal packing diagram viewed along $c$-axis. Hydrogen bonds are depicted as the dash lines.

Figure 3: Oscilloscope trace of SHG signals of DAST, HMQ-CBS (I) and HMQ-CBS (II), respectively. 


\section{Figure Content:}
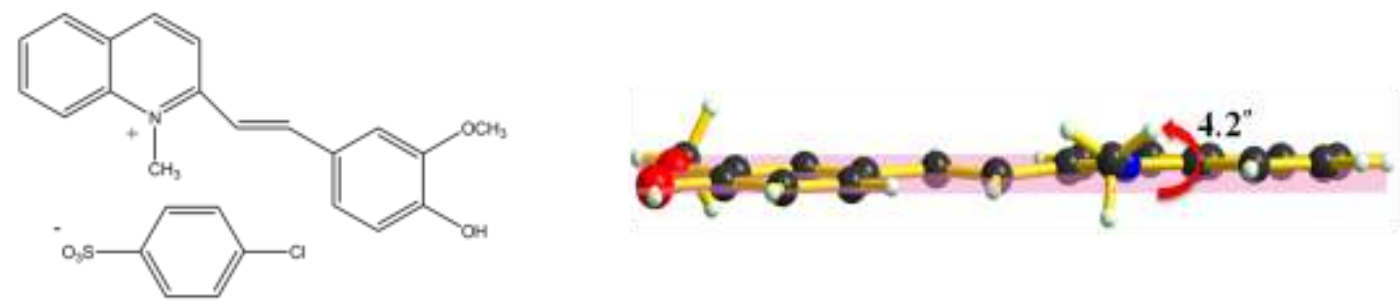

(a)

(b)
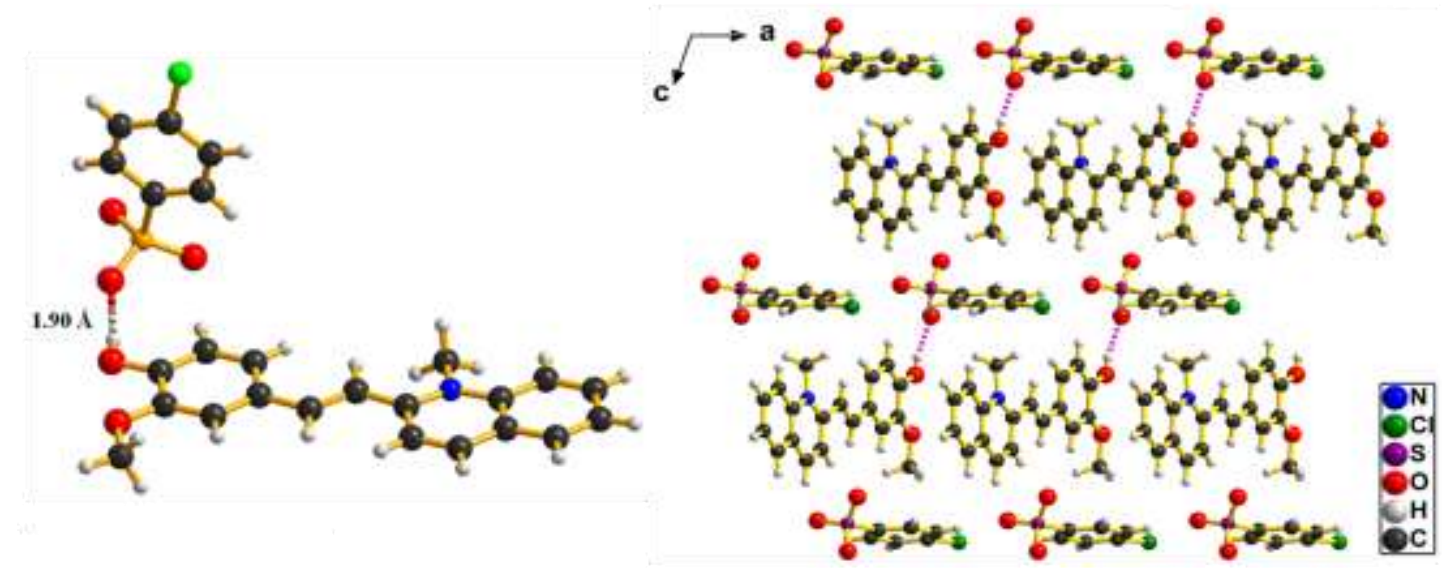

(c)

(d)

Fig. 1. Scheme and crystal structures of HMQ-CBS in phase-I. (a) schematic diagram of HMQ-CBS; (b) molecular conformation of the cationic moiety; (c) molecular structure; and (d) crystal packing diagram viewed along $b$-axis. Hydrogen bonds are depicted as the dash lines. 
(a)

(b)
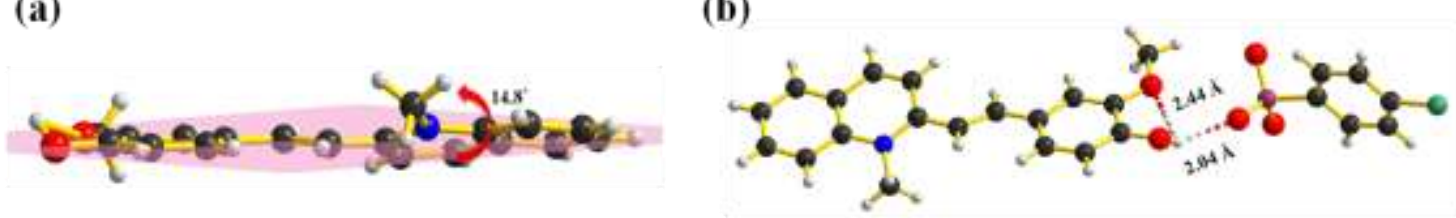

(c)

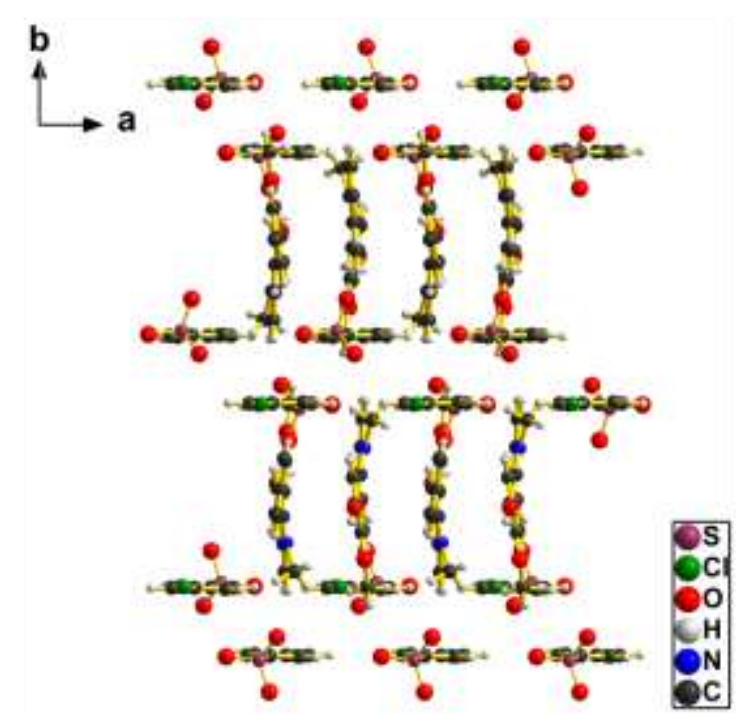

Fig. 2. Crystal structures of HMQ-CBS in phase-II. (a) molecular conformation of the cationic moiety; (b) molecular structure, and (c) crystal packing diagram viewed along $c$-axis. Hydrogen bonds are depicted as the dash lines. 


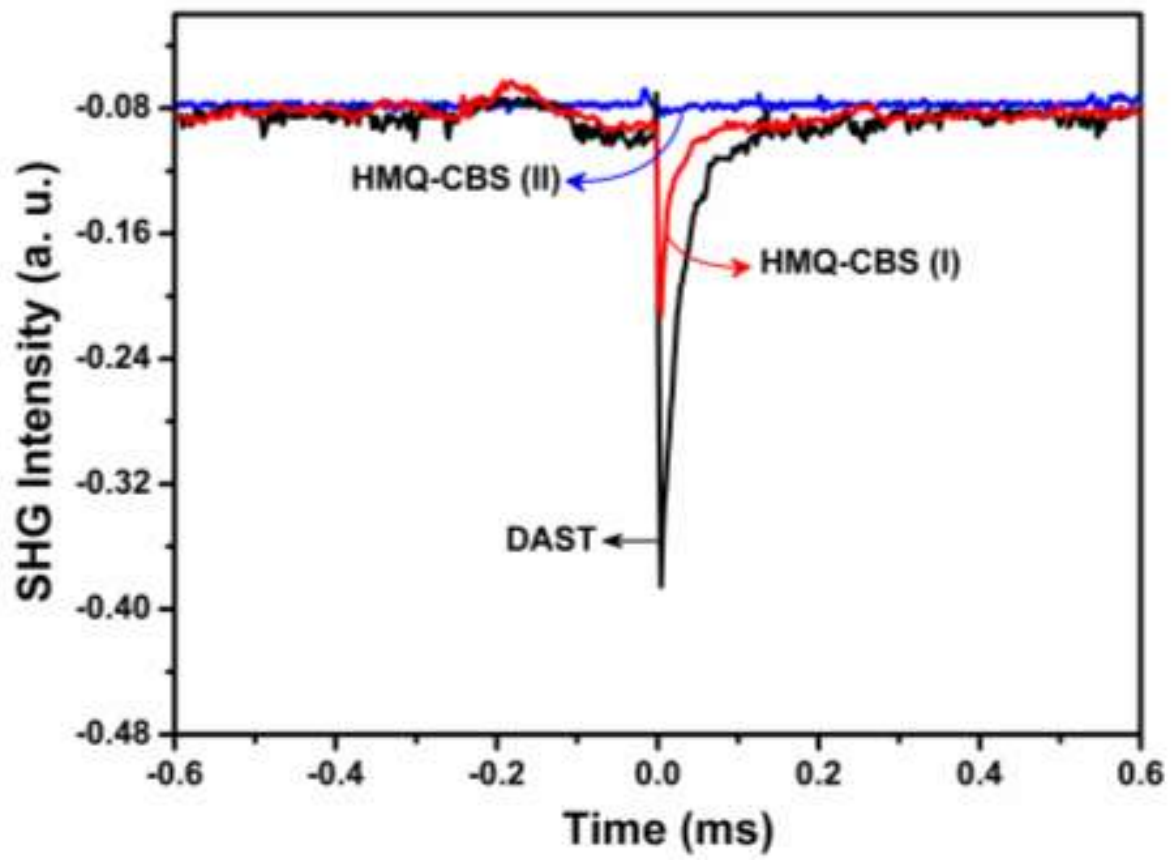

Fig. 3. Oscilloscope traces of the SHG signals for DAST, HMQ-CBS (I) and HMQ-CBS (II), respectively. 

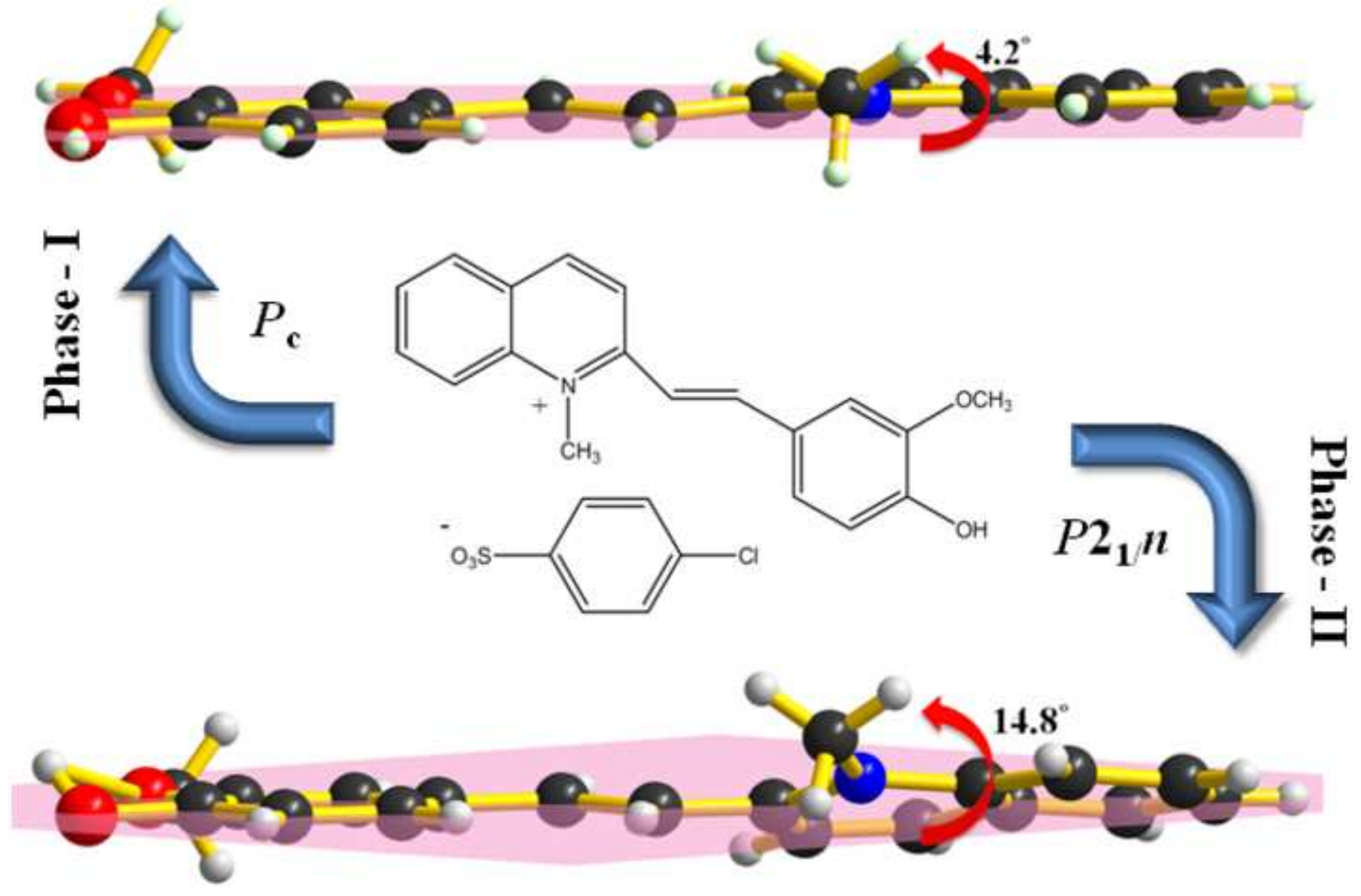